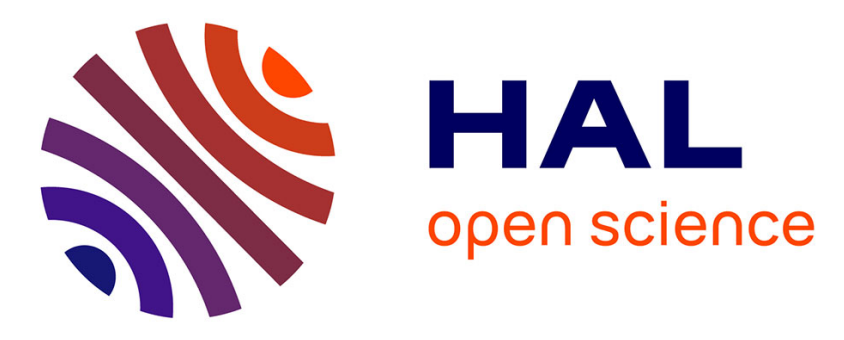

\title{
Prenatal exposure to the contaminant perfluorooctane sulfonate elevates lipid peroxidation during mouse fetal development but not in the pregnant dam
}

\author{
Yiu Yiu Lee, C. K C Wong, Camille Oger, Thierry Durand, Jean-Marie \\ Galano, Jetty Chung-Yung Lee
}

\section{To cite this version:}

Yiu Yiu Lee, C. K C Wong, Camille Oger, Thierry Durand, Jean-Marie Galano, et al.. Prenatal exposure to the contaminant perfluorooctane sulfonate elevates lipid peroxidation during mouse fetal development but not in the pregnant dam. Free Radical Research, 2015, 49 (8), pp.1015-1025. 10.3109/10715762.2015.1027199 . hal-02576249

\section{HAL Id: hal-02576249 \\ https://hal.science/hal-02576249}

Submitted on 14 May 2020

HAL is a multi-disciplinary open access archive for the deposit and dissemination of scientific research documents, whether they are published or not. The documents may come from teaching and research institutions in France or abroad, or from public or private research centers.
L'archive ouverte pluridisciplinaire HAL, est destinée au dépôt et à la diffusion de documents scientifiques de niveau recherche, publiés ou non, émanant des établissements d'enseignement et de recherche français ou étrangers, des laboratoires publics ou privés. 



\title{
Prenatal exposure to the contaminant perfluorooctane sulfonate elevates lipid peroxidation during mouse fetal development but not in the pregnant dam
}

\author{
Y. Y. Lee ${ }^{1}$, C. K. C. Wong ${ }^{2}$, C. Oger ${ }^{3}$, T. Durand ${ }^{3}$, J.-M. Galano ${ }^{3}$ \& J. C.-Y. Lee ${ }^{1}$ \\ ${ }^{1}$ School of Biological Sciences, The University of Hong Kong, Hong Kong SAR, ${ }^{2}$ Partner State Key Laboratory of Environmental and \\ Biological Analysis, Department of Biology, Croucher Institute for Environmental Sciences, Hong Kong Baptist University, Hong Kong \\ SAR, and ${ }^{3}$ Institut des Biomolécules Max Mousseron, Université de Montpellier, France
}

\begin{abstract}
Perfluorooctane sulfonate (PFOS), a member of the perfluorinated chemical family, has been convincingly demonstrated to affect lipid metabolism in animals and humans and readily crosses the placenta to exert its effects on the developing fetuses. While its exact mechanism is still not clear, PFOS exposure has long been suggested to exert its toxicity via oxidative stress and/or altered gene expression. Levels of PFOS and malondialdehyde in various organs and cell cultures have been widely determined as general indicators of non-specific lipid peroxidation after PFOS exposure. In this study, the oxidation of precise polyunsaturated fatty acids and their metabolites, derived from enzymatic and non-enzymatic pathways was determined following PFOS exposure in both adult and maternal/fetal mice. CD-1 mice were exposed to $3 \mathrm{mg} / \mathrm{kg}$ body weight/day of PFOS in corn oil by oral gavage until late gestation (GD17). We demonstrated that lipid peroxidation was particularly and exclusively affected in fetuses exposed to PFOS, but this was not the case in the maternal mice, where limited effects were observed in the enzymatic oxidation pathway. In this study, we demonstrated that PFOS-induced lipid peroxidation might have a greater impact in free radical generation in fetuses than in dams and could be responsible for affecting fetal development. In addition, antioxidant enzymes, such as superoxide dismutase and catalase, appeared to maintain oxidative stress homeostasis partially in adult mice exposed to PFOS. Taken together, our results might elucidate the mechanism of how PFOS induces oxidative stress in vivo.
\end{abstract}

Keywords: PFOS, isoprostanoids, isofuranoids, oxidative stress, PUFA

\begin{abstract}
Abbreviations: 4(RS)-4- $\mathrm{F}_{4 \mathrm{t}}-\mathrm{NeuroP}, 4(R S)-4-\mathrm{F}_{4 \mathrm{t}}-$ neuroprostane; 5- $\mathrm{F}_{2 \mathrm{t}}-\mathrm{IsoP}, 5-\mathrm{F}_{2 \mathrm{t}}$-isoprostane; 8- $\mathrm{F}_{3 \mathrm{t}}-\mathrm{IsoP}_{\text {s }}$ 8- $\mathrm{F}_{3 \mathrm{t}}$ - $\mathrm{isoprostane}$; $15-\mathrm{F}_{2 \mathrm{t}}-\mathrm{IsoP}, 15-$

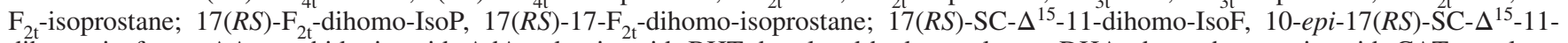
dihomo-isofuran; AA, arachidonic acid; AdA, adrenic acid; BHT, butylated hydroxytoluene; DHA, docosahexaenoic acid; CAT, catalase; CYP450, cytochrome P450; EPA, eicosapentaenoic acid; $\mathrm{F}_{2}$-IsoP, $\mathrm{F}_{2}$-isoprostane; HETE, hydroxyeicosatetraenoic acid; IsoFs, isofurans; LC-MS/MS, liquid chromatography-tandem mass spectrometry; LOX, lipoxygenase; MTBE, methyl-tert-butyl ether; NeuroFs, neurofurans; PFOS, perfluorooctane sulfonate; PUFA, polyunsaturated fatty acid; SOD, superoxide dismutase
\end{abstract}

\section{Introduction}

Perfluorooctane sulfonate (PFOS, $\mathrm{C}_{8} \mathrm{~F}_{17} \mathrm{SO}_{3} \mathrm{H}$ ) is a member of the perfluorinated chemical family and is synthetically produced for industrial use. The stable carbon-fluorine bonds of the compound make it highly resistant to all forms of degradation and exhibit environmental persistence. Due to their unique chemical properties of water and oil repulsion and an improved reduction in surface tension compared with those of other surfactants, they are mainly used as multi-resistant coatings for a wide range of products, including paper, textile, and food packaging [1]. The extensive use of PFOS since the 1950s has resulted in substantial bioaccumulation; a wide distribution in human [2], wildlife [3], arctic populations [4], and detrimental effects to growth and development.

The main route of PFOS exposure in humans is believed to be through the consumption of contaminated water and food. Traces of PFOS have been found in human plasma, umbilical cord blood, breast milk, and liver. The half-life of PFOS elimination is estimated to be approximately 4-5 years for humans [2], 1-2 months for rodents, and 4 months for monkeys [5]. The proposed mechanism of PFOS toxicity is commonly related to the generation of reactive oxygen species (ROS) and the induction of oxidative stress, but its exact mode of action remains largely unknown. Because of the potential adverse health effects in humans, and its persistence and toxicity in animal models, companies have voluntarily stopped industrial PFOS production in most countries since 2000 . However, due to its demand in the market, enterprises in Asia began using PFOS and its precursor in production, which has dramatically increased since 2003 [6]. Therefore, the reinstatement of PFOS production continues to threaten human health globally. In 2009, PFOS was listed as one of the most concerning persistent organic pollutants at the Stockholm Convention and restrictions have been sought on its production and use.

Correspondence: Jetty Chung-Yung Lee, School of Biological Sciences, Kadoorie Biological Sciences Building, The University of Hong Kong, Pokfulam Road, Hong Kong SAR. Tel: + 852 22990318. Fax: + 852 25599114. E-mail: jettylee@hku.hk 
PFOS has also been shown to cross the placenta and enter fetal circulation to affect early development by disrupting thyroid hormone homeostasis [7], glucose metabolism [8], and lipid metabolism $[9,10]$. Maternal exposure to PFOS results in fetal brain accumulation during animal gestation [11]. PFOS causes peroxisome proliferation, and affects the enzymes and proteins that are involved in lipid metabolism, leading to metabolic disruption [12]. However, studies in laboratory rodents have primarily assessed blood lipid levels, including triglycerides, low-density lipoprotein, and high-density lipoprotein $[9,10,13]$, while studies in cultured cells [14] and fish embryos [15] have studied overall lipid peroxidation in the form of malondialdehyde (MDA). There are insufficient reports on the different classes and species of lipids and their oxidized products after PFOS exposure.

In the present study, we examined the levels of different lipid precursors (focusing on polyunsaturated fatty acids (PUFAs)) and their oxidized metabolites in adult, maternal, and fetal mouse organs in the presence of PFOS. Our findings help to elucidate the mechanism of PFOS-induced oxidative stress, which leads to detrimental effects on health and development.

\section{Materials and methods}

\section{Animal handling}

All animals were maintained and used in accordance with the guidelines of the Department of Health, the Government of Hong Kong Special Administrative Region. Experimental procedures were performed in accordance with the guidelines of Hong Kong Baptist University Committee on the Use of Human and Animal Subjects (Permit no. 261812). Female CD-1 mice (6-8 weeks old) were purchased from the Laboratory Animal Service Centre of the Chinese University of Hong Kong. The mice were acclimated for one week before the experiments began. The conditions and well-being of the animals were closely monitored, and no death was recorded during the study. Mice were mated overnight and vaginal plugs were identified the next day and counted as day 1 of gestation (GD1). Pregnant mice were randomized into two groups, control and PFOS treatment ( $3 \mathrm{mg} / \mathrm{kg}$ b.w.) groups, with $n \geq 4$ per group. The dose of $3 \mathrm{mg} / \mathrm{kg}$ was chosen based on a dose-dependent PFOS toxicity study, which showed that rats treated with $3 \mathrm{mg} / \mathrm{kg}$ exhibited significant PFOS toxicity with moderate rate of mortality [16]. They were housed in polypropylene cages with sterilized bedding, under controlled temperature $\left(20-25^{\circ} \mathrm{C}\right)$ and humidity $(35-70 \%)$ with 12 -h alternating light and dark cycles, and fed with standard lab chow (LabDiet 5001 Rodents Diet) and water ad libitum. Oral PFOS (98\% purity, Sigma Aldrich) doses was prepared by dissolving the PFOS in dimethyl sulfoxide (DMSO; final concentration, $\leq 0.05 \%$ ) and subsequently mixing it with corn oil. The control group was given corn oil with $0.05 \%$ DMSO. Both the control and treatment groups were fed daily via oral gavage until GD17. In a separate study, mature (non-pregnant) female mice were housed in the same setting and given PFOS (3 mg/kg b.w.) for 14 days.

\section{Tissue collection and PFOS measurement}

Maternal mice $\left(\mathrm{F}_{0}\right)$ were euthanized via cervical dislocation, and the unborn fetuses $\left(\mathrm{F}_{1}\right)$ were surgically harvested on GD17. Maternal blood was collected via cardiac puncture. Blood was allowed to clot and centrifuged at $3000 \times \mathrm{g}$ for $15 \mathrm{~min}$ at $4^{\circ} \mathrm{C}$ to produce a serum sample. The collected serum was immediately stored at $-20^{\circ} \mathrm{C}$ until analysis. Maternal (liver and placenta) and fetal organs (liver, brain, and kidney) were harvested and washed with phosphate-buffered saline containing $0.005 \%(\mathrm{w} / \mathrm{v})$ butylated hydroxytoluene (BHT) and $0.005 \%(\mathrm{w} / \mathrm{v})$ indomethacin. The harvested organs were snap frozen and stored at $-80^{\circ} \mathrm{C}$ until further analysis.

PFOS concentrations in maternal sera were measured as described previously [17]. In brief, the serum sample was diluted and mixed with tetra-n-butyl ammonium hydrogen sulfate buffer and methyl-tert-butyl ether (MTBE). The organic phase was transferred, re-extracted with MTBE, and pooled. After drying under a stream of nitrogen, it was reconstituted with $10 \mathrm{mM}$ ammonium acetate:acetonitrile $(6: 4, \mathrm{v} / \mathrm{v})$ and analyzed by liquid chromatography-tandem mass spectrometry (LC-MS/MS, Agilent 1200 series, Agilent Technologies, USA). PFOS and labeled PFOS standards used for calibration were both prepared in methanol. A guard column (Zorbax Eclipse PlusC8, Agilent Technologies, USA) connected to Zorbax Eclipse Plus C8 column $(100 \mathrm{~mm} \times 2.1 \mathrm{~mm}, 3.5 \mu \mathrm{m}$, Agilent Technologies, USA) was established according to the procedure described by Zhao et al. [18] and used for the analysis.

\section{Tissue lipid extraction}

Tissue homogenization and extraction were performed as described previously [19] with modifications. Briefly, tissue samples were homogenized in ice-cold Folch solution (chloroform:methanol, 2:1, v/v) with $0.01 \%(\mathrm{w} / \mathrm{v})$ BHT. Aqueous $0.9 \% \mathrm{NaCl}$ was added to allow phase separation. After centrifugation at $800 \times \mathrm{g}$ for $10 \mathrm{~min}$, at $4^{\circ} \mathrm{C}$, the lower phase was transferred to a glass vial and the solvent was evaporated under a stream of nitrogen gas. The dried Folch extract was reconstituted with methanol and hydrolyzed overnight in the dark at room temperature with $1 \mathrm{M}$ aqueous potassium hydroxide $(1: 1, \mathrm{v} / \mathrm{v})$. After hydrolysis, the sample was neutralized and formic acid ( $\mathrm{pH}: 4.5)$ was added [20]. The lipid extract mixtures were centrifuged to remove any protein precipitate before solid-phase extraction. For solid-phase extraction, 60-mg MAX columns (Oasis, Waters, USA) were used to purify the hydrolyzed samples according to the method described by Lee et al. [21].

\section{Lipid analysis}

Oxidized lipid product and fatty acid measurements [22] were performed using LC-MS/MS (1290 Infinity, Agilent 
Technologies and Sciex 3200 QTRAP, Applied Biosystems, USA). A Kinetex HILIC column $(30 \times 3 \mathrm{~mm}, 2.6$ $\mu \mathrm{m}$, Phenomenex, USA) was used and maintained at room temperature. The mobile phase consisted of $0.1 \%$ acetic acid in water (A) and $0.1 \%$ acetic acid in methanol (B). A step-wise gradient elution from $60 \% \mathrm{~B}$ for $5 \mathrm{~min}$, followed by increasing to $70 \%$ for $1 \mathrm{~min}$, and $100 \%$ for a final $1 \mathrm{~min}$ was used. The flow rate was set to $100 \mu 1 / \mathrm{min}$ for the first $5 \mathrm{~min}$, and subsequently to $200 \mu \mathrm{l} / \mathrm{min}$. The sample injection volume was $20 \mu \mathrm{l}$. The MS/MS was operated in the electrospray ionization (ESI) negative mode with a source temperature of $500^{\circ} \mathrm{C}$ and capillary temperature of $250^{\circ} \mathrm{C}$. The collision energy was optimized to maximize the ion currents of the precursor to produce ion dissociation. The analytes were detected by MS/MS using multiple reactions monitoring (MRM) and their mass-to-charge ratio $(\mathrm{m} / \mathrm{z}$ ) was assessed according to Supplementary Table I (to be found at online http://informahealthcare.com/doi/abs/ 10.3109/10715762.2015.1027199). Quantitation of each analyte was achieved by relating the peak area with its corresponding heavy labeled internal standard peak. Where available, heavy labeled isotope was used for quantitation, whereas for those without heavy labeled isotope, quantitation was achieved by relating to deuterated standards with the closest chemical structure. All lipid standards were purchased from Cayman Chemical (Ann Arbor, MI, USA) or synthesized by Institut des Biomolécules Max Mousseron (IBMM, Montpellier, France).

\section{Cholesterol analysis}

Cholesterol analysis was performed using the same setup for lipids with different buffers and the ESI mode [22]. The LC mobile phase of $5 \mathrm{mM}$ ammonium acetate in $10 \%$ methanol (A) and $5 \mathrm{mM}$ ammonium acetate in $85 \%$ methanol (B) was set to a constant $200 \mu \mathrm{l} / \mathrm{min}$ flow rate. The mobile phase ran at a constant of $0 \% \mathrm{~B}$ phase from 0 to $4 \mathrm{~min}$ and gradually increased to $100 \%$ from 4 to $5 \mathrm{~min}$, and then $100 \%$ $\mathrm{B}$ for a subsequent $2 \mathrm{~min}$. The sample injection volume was $20 \mu \mathrm{l}$. An ESI positive mode was applied for QTRAP 3200 triple quadrupole MS (Sciex Applied Biosystems, USA) with a source temperature of $400^{\circ} \mathrm{C}$ and capillary temperature of $250^{\circ} \mathrm{C}$. The analytes were detected by MS/MS using MRM and the transitions were monitored as suggested by Shui et al. [23]. Cholesterol quantitation was achieved by relating the peak area with its corresponding internal standard peak. Both cholesterol and deuterated cholesterol standards were purchased from Avanti Polar Lipids (USA).

\section{Antioxidant enzyme activity assays}

Superoxide dismutase (SOD) and catalase (CAT) enzyme activities were analyzed using commercial kits from Cayman Chemical (Ann Arbor, MI, USA) according to the manufacture instructions. Briefly, samples were homogenized and centrifuged, and the supernatants were removed for immediate analysis. Total protein concentrations in each supernatant were measured by the Bradford assay (Bio-Rad, USA) and enzyme activities were normalized to per $1 \mathrm{mg}$ of total protein. One unit of SOD activity was defined as "the amount of enzyme needed to exhibit 50\% dismutation of the superoxide radical." One unit of catalase activity was defined as "the amount of enzyme that will cause the formation of $1.0 \mathrm{nmol}$ of formaldehyde per minute at $25^{\circ} \mathrm{C}$."

\section{Statistical analysis}

Statistical analysis was performed and figures were prepared using GraphPad Prism version 6.0 (GraphPad Software, CA, USA). All values were expressed as the mean \pm standard deviation (SD). Data were analyzed by Student's t-test and a $p$ value of $<0.05$ was denoted as statistically significant.

\section{Results}

Fetuses mandatorily received PFOS from dams via placenta

In the study, prolonged PFOS exposure via oral administration markedly increased PFOS levels in the serum of CD-1 maternal mice (Figure 1$)$ and was significantly $(p<0.0001)$ higher than that in the control group. The PFOS concentration was $41.98 \pm 8.20 \mu \mathrm{g} / \mathrm{ml}$ in the serum of maternal mice after 16 days of treatment during pregnancy, which was approximately 175 -fold higher than that in the control group. This confirms that PFOS is delivered and retained in the body of treated pregnant mice for distribution to other organs. Due to the limited fetal blood specimens present, PFOS measurements in fetal serum were not obtained, but the transfer of PFOS from the maternal blood circulation to the fetus through the placenta is thought to be the only way for the fetus to be exposed to PFOS during gestation.

\section{PFOS altered cholesterol and PUFA levels in the exposed fetus}

To determine the effects of PFOS on lipid metabolism, endogenous levels of PUFAs and cholesterol were

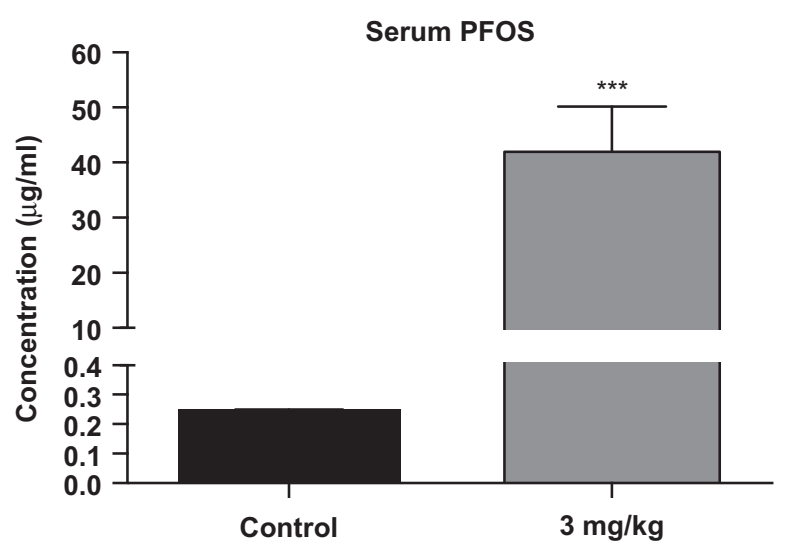

Figure 1. PFOS concentrations in maternal serum. Values in each column are expressed as the mean $\pm \mathrm{SD}$. The number of sample replicates of maternal serum in the control group is $n=4$, and in the $3 \mathrm{mg} / \mathrm{kg}$ PFOS group it is $n=4$. ${ }^{* * *} p<0.001$ compared with control. 

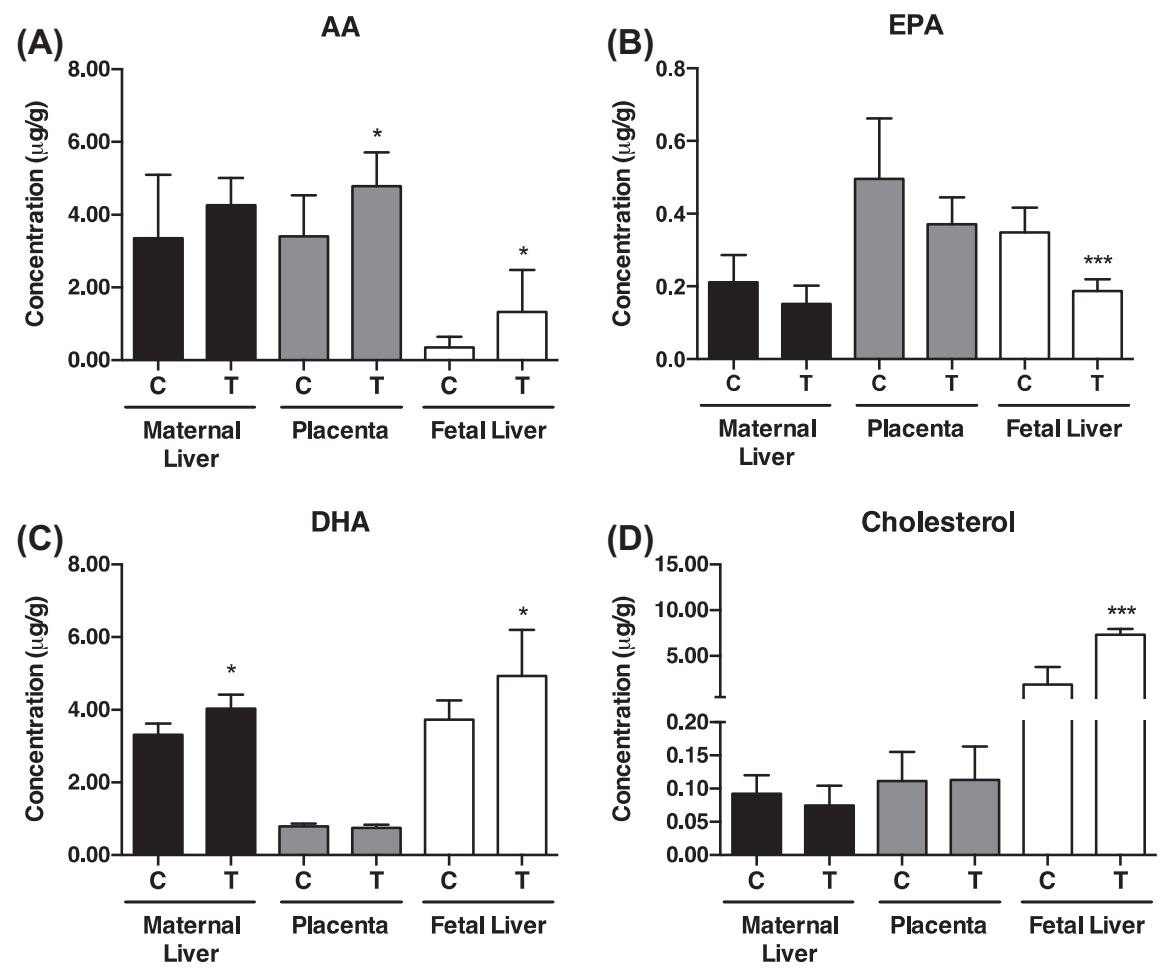

Figure 2. Concentrations of AA, EPA, DHA, and cholesterol in maternal and fetal livers and placenta. Panels represent AA (A), EPA (B), DHA (C), and cholesterol (D). Values in each column are expressed as the mean \pm SD. C: control; T: PFOS-treated. The number of sample replicates of maternal liver is $n=4$, and that of placenta and fetal liver is $n=8{ }^{*} p<0.05, * * * p<0.001$ compared with control.

measured in both the control and PFOS-treated groups (Figure 2A-D). The organs were chosen (maternal liver, placenta, and fetal liver) based on the route of PFOS exposure. In the present study, we quantified cholesterol and PUFA precursors, including arachidonic (AA, 20:4 $n$-6), eicosapentaenoic (EPA, 20:5 n-3), and docosahexaenoic (DHA, 22:6n-3) acids. The levels of cholesterol and PUFA precursors in PFOS-treated fetal livers were consistently different from those in the control, with significant increases in AA, DHA $(p<0.05)$, and cholesterol $(p<0.001)$, while EPA decreased significantly $(p<0.001)$. However, DHA in maternal liver and AA in placenta were both elevated $(p<0.05)$ after PFOS treatment. No significant changes were observed in other tissues after PFOS treatment compared with those in the control group.

\section{PFOS induced lipid peroxidation primarily in the fetus}

To probe the pathway in which PFOS participates to induce oxidative stress, non-enzymatic (Figure $3 \mathrm{~A}-\mathrm{F}$ ) and enzymatic (Figure 4A-H) oxidized lipid mediators were quantified. Non-enzymatic oxidized lipid products were measured, namely $15-\mathrm{F}_{2 \mathrm{t}^{-}}$isoprostane (IsoP) and $5-\mathrm{F}_{2 \mathrm{t}^{-}}$ IsoP derived from AA, $8-\mathrm{F}_{3 \mathrm{t}}$-IsoP liberated from EPA, and $4(R S)-\mathrm{F}_{4 \mathrm{t}}$-neuroprostance (NeuroP) released from DHA, as well as 7 $\beta$-hydroxycholesterol and 7-ketocholesterol produced from cholesterol. Collectively, non-enzymatic oxidized lipid products were significantly increased in the liver of PFOS-treated fetuses, but not in maternal liver and placenta. This indicates that PFOS treatment enhanced lipid peroxidation in developing fetuses, regardless of the precursor concentration.

We also investigated enzymatically oxidized lipid products derived from the lipoxygenase (LOX) and cytochrome P450 (CYP450) pathways. Concentrations of the various hydroxyeicosatetraenoic acids (HETEs, derived from AA) released via the LOX (Figure 4A-D) and CYP450 pathways (Figure $4 \mathrm{E}-\mathrm{H}$ ) showed no differences between the two groups, except for a decrease in the 15(S)-HETE level in fetal liver and an increased 5(S)-HETE level in maternal liver in the PFOS group compared with those in the control. The concentration of 27-hydroxycholesterol was also increased in the fetal liver of the PFOS group compared with that in the control. The levels of oxidized lipid products generated by the enzymatic pathway are, therefore, inconsistent compared with the non-enzymatic pathway, showing the latter to be predominant in PFOS-associated lipid peroxidation.

\section{Maternal antioxidant enzyme activities coped with PFOS-induced oxidative stress}

Despite the substantial amount of PFOS that is delivered and retained in the maternal body, our data showed that maternal mice coped with the oxidative stress better than fetuses (Figures 3 and 4). We hypothesized that the welldeveloped antioxidant enzyme system in the dam is the reason for this difference. Thus, we assessed the activities of the antioxidant enzymes SOD and CAT (Figure 5A-B). However, due to the limitation of organ samples, fetal livers were not available for these measurements. When 

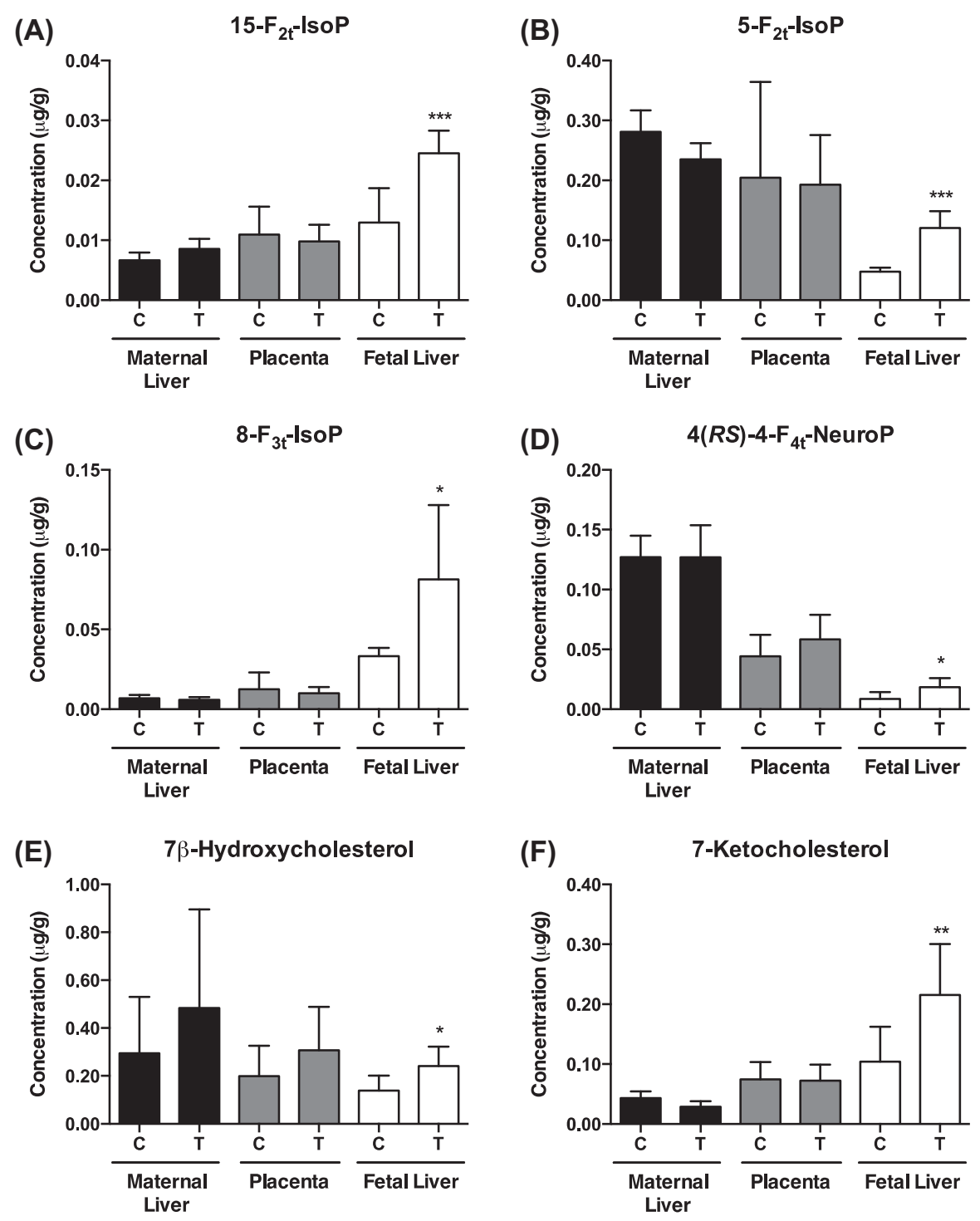

Figure 3. Non-enzymatically oxidized lipid products of PUFAs after PFOS exposure. Panels represent $\mathrm{F}_{2}$-IsoPs (A-B) derived from AA, $\mathrm{F}_{3}$-IsoP (C) derived from EPA, $\mathrm{F}_{4}$-NeuroP (D) derived from DHA, and 7 $\beta$-hydroxycholesterol (E) and 7-ketocholesterol (F) derived from cholesterol. Values in each column are expressed as the mean \pm SD. C: control; T: PFOS-treated. The number of sample replicates of maternal liver is $n=4$, and that of placenta and fetal liver is $n=8 .{ }^{*} p<0.05,{ }^{* *} p<0.001$ compared with control.

evaluating placental antioxidant enzyme activities, we found that the activities of SOD and CAT in PFOS-treated maternal liver and placenta were not significantly different from those of the control.

\section{Lipid peroxidation in fetal brain is in lesser extent than that in liver and kidney}

To further evaluate the effects of PFOS-induced lipid peroxidation (focusing on PUFAs) in other vital organs such as brain and kidney, we conducted a separate experiment where the adult (non-pregnant) female mice and unpaired fetuses were exposed to the same dose of PFOS for a similar duration (fetuses: 17 days; adult: 14 days), and their levels of lipid precursors and oxidized products were measured. The lipid precursors analyzed in this study include AA, adrenic acid (AdA, 22:4, $n$-6; an elongated form of AA), EPA, and DHA. Various isoprostanoids and isofuranoids (generated under higher oxygen tension) derived from different precursors were determined to assess their levels of lipid peroxidation after PFOS exposure.

Table I illustrates the levels of lipid precursors and their mediators from adult and fetal mouse brain tissues. The PFOS-treated brain tissues showed significant increases in AA $(p<0.05)$ and AdA $(p<0.01)$ but not in EPA and DHA, while the levels in fetal brain were not altered compared with those in the control group. Non-enzymatic oxidized lipid products in both adult and fetal brain tissues were not statistically significant between the two groups, except for the increase in neurofuran and $8-\mathrm{F}_{3 \mathrm{t}}$-IsoPs $(p<0.05)$ in PFOS-treated adult and fetal tissues, respectively. Similarly, enzymatically oxidized lipid products derived from the LOX and CYP450 pathways were quantified in adult and fetal brain tissues (Table II). These products in PFOS-exposed adult mice brain tissues were not affected compared with those in control, but the fetal counterparts for $9(S)$-HETE, 5(S)-HETE, and 8(S)-HETE production were significantly inhibited. 

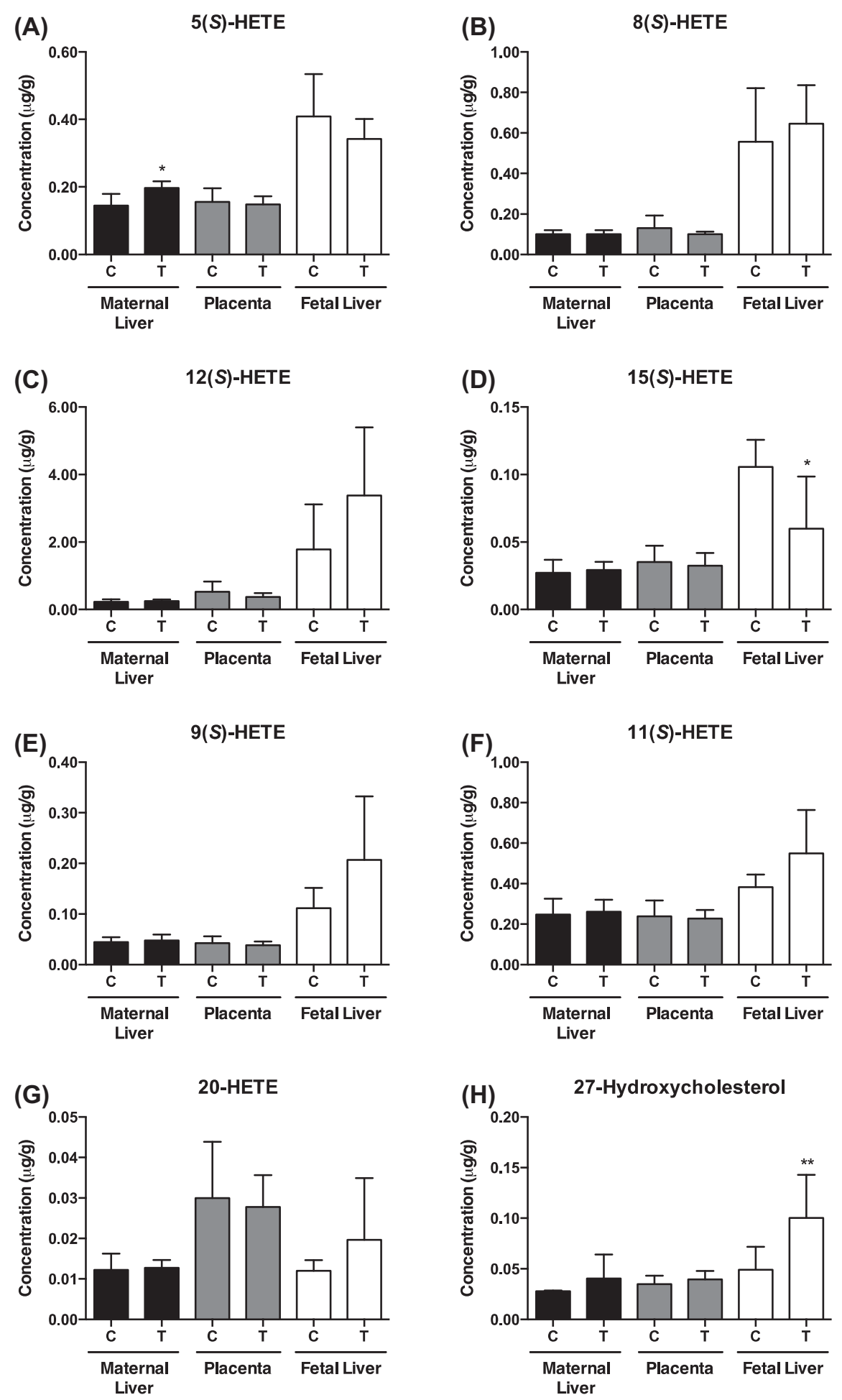

Figure 4. Enzymatically oxidized lipid products of AA and cholesterol. Panels represent products from the LOX (A-E) and CYP450 (F-H) pathways. Values in each column are expressed as the mean \pm SD. C: control; T: PFOS-treated. The number of sample replicates of maternal liver is $n=4$, and that of placenta and fetal liver is $n=8 .{ }^{*} p<0.05$ compared with control.

Identical sets of lipid precursors and oxidized lipid mediators were analyzed for adult and fetal kidneys (Table III). Seemingly, PFOS exposure appeared to have an apparent effect on both adult and fetal kidney lipid peroxidation. While the levels of all lipid precursors (except for DHA) and their non-enzymatic metabolites in the PFOS-treated fetal kidney increased compared with those in the control, lipid precursors and oxidized products in
PFOS-treated adult kidney showed a significant decrease

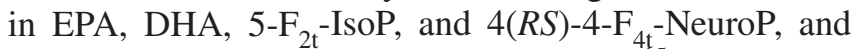
$17(R S)-\mathrm{F}_{2 \mathrm{t}}$-dihomo-IsoP and 17(RS)-SC- $\Delta^{15}$-11-dihomoIsoF from $\mathrm{AdA}$, but $15-\mathrm{F}_{2 \mathrm{t}}$-IsoP (an overall in vivo oxidative stress biomarker) was elevated.

Finally, enzymatically oxidized lipid products in PFOStreated adult mice kidney had reduced $9(S)-, 11(S)$-, $8(S)$-, and 12(S)-HETE levels, whereas 5(S)- and 15(S)-HETE 

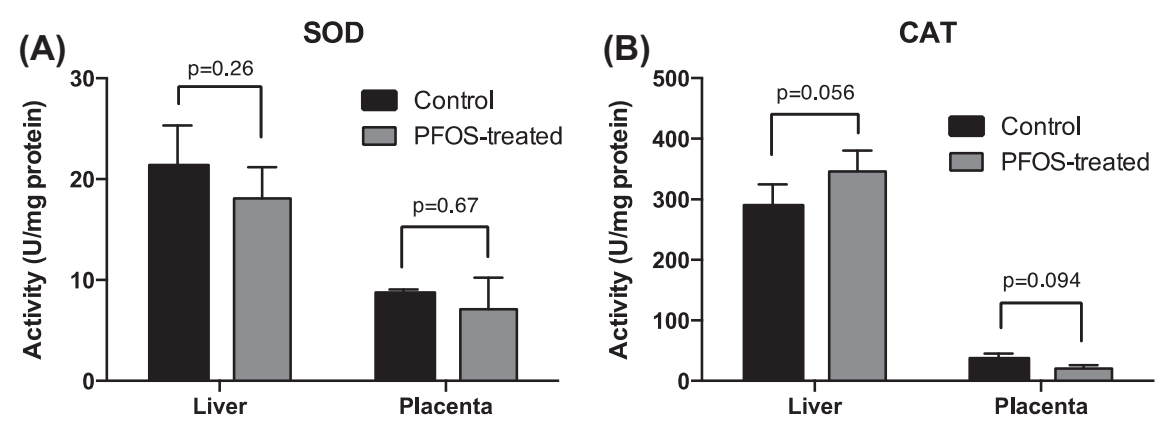

Figure 5. Antioxidant enzyme activities in maternal liver and placenta. Panels represent SOD (A) and CAT (B) enzyme activities. Enzyme activities are expressed as unit per $\mathrm{mg}$ of total protein. Values in each column are expressed as the mean \pm SD. The number of sample replicates of maternal liver and placenta is $n=4$.

levels were increased compared with those in the control (Table IV). PFOS-treated fetal kidney showed a decreased amount of 5(S)- and 11(S)-HETEs, while 15(S)-HETE levels increased compared with the control group. Aside from these, other precursors and oxidized lipid products (Table IV) were not significantly altered between the control and PFOS-treated groups.

\section{Discussion}

The purpose of this study was to probe how PFOS affects lipid metabolism and induces oxidative stress by evaluating the levels of oxidized PUFAs and cholesterol products from enzymatic and non-enzymatic pathways. It is well documented that the elevation of these oxidized lipid products in vivo leads to metabolic and cognitive dysfunction and a risk of developing cardiovascular disease, diabetes, cancer, and neurodegeneration [21,24-26].

In this study, we found that lipid precursor levels in PFOS-treated fetal, but not maternal livers were consistently elevated, with the exception of EPA. As reported by others, the increase in these lipid precursor levels is rationalized by the reduction in serum lipid levels, in which triglycerides and cholesterols are transported into the liver from the circulation and retained, leading to lipid vacuolation $[27,28]$. This is due to the upregulation of hepatic lipoprotein lipase and fatty acid translocase for lipoprotein trafficking, and the uptake of free fatty acids with a simultaneous reduction in apolipoprotein-B100 level that is needed for very-low-density lipoprotein packaging and secretion $[9,10]$. However, we noted a significant reduction in EPA level in fetal liver, which may indicate a possible modification of the enzymes that participate in the bioconversion of EPA to docosapentaenoic acid (DPA, 22:5 n-3) and DHA, such as $\Delta^{5}$ - and $\Delta^{6}$-desaturases [29], in the presence of PFOS.

Based on previous findings, prenatal exposure to PFOS in humans and rodents was approximately twice as much as what is present in the fetus, whether harvested from plasma or postnatal liver [30,31]. On average, mouse gestation is approximately 18 days, and the fetal samples used

Table I. Level of PUFAs and their non-enzymatic oxidized lipid products in adult and fetal mice brain after PFOS treatment.

\begin{tabular}{|c|c|c|c|c|}
\hline & \multicolumn{4}{|c|}{ Brain } \\
\hline & \multicolumn{2}{|c|}{ Adult } & \multicolumn{2}{|c|}{ Fetus } \\
\hline & Control & PFOS-treated & Control & PFOS-treated \\
\hline \multicolumn{5}{|l|}{ Lipia precursors } \\
\hline $\mathrm{AA}^{+}$ & $4.27 \pm 2.13$ & $9.3 \pm 1.04 *$ & $4.60 \pm 0.48$ & $5.10 \pm 0.60$ \\
\hline AdA & $75.18 \pm 13.40$ & $147.15 \pm 5.61^{* *}$ & $82.55 \pm 8.86$ & $85.72 \pm 9.70$ \\
\hline EPA & $6.96 \pm 0.38$ & $8.69 \pm 1.38$ & $47.15 \pm 14.69$ & $45.27 \pm 15.59$ \\
\hline DHA & $459.46 \pm 96.62$ & $556.00 \pm 69.78$ & $551.33 \pm 83.53$ & $492.43 \pm 75.57$ \\
\hline \multicolumn{5}{|l|}{ Isoprostanoids } \\
\hline $15-\mathrm{F}_{2 \mathrm{t}}$-IsoP & $9.58 \pm 2.67$ & $9.56 \pm 3.35$ & $1.50 \pm 0.42$ & $1.38 \pm 0.28$ \\
\hline $5-\mathrm{F}_{2 \mathrm{t}}-\mathrm{IsoP}$ & $31.34 \pm 13.65$ & $57.18 \pm 62.87$ & $6.95 \pm 1.26$ & $5.77 \pm 2.76$ \\
\hline $8-\mathrm{F}_{3 \mathrm{t}}$-IsoP & $9.80 \pm 0.30$ & $7.90 \pm 1.68$ & $0.61 \pm 0.12$ & $0.80 \pm 0.21^{*}$ \\
\hline $4(R S)-4-\mathrm{F}_{4 \mathrm{t}}-\mathrm{NeuroP}$ & $37.64 \pm 17.74$ & $34.81 \pm 9.79$ & $32.06 \pm 7.79$ & $31.21 \pm 7.68$ \\
\hline $17(R S)-\mathrm{F}_{2 \mathrm{t}}$-dihomo-IsoP & $14.35 \pm 3.04$ & $18.14 \pm 12.50$ & $2.67 \pm 0.71$ & $2.59 \pm 0.42$ \\
\hline \multicolumn{5}{|l|}{ Isofuranoids } \\
\hline IsoF & $3.73 \pm 1.30$ & $5.50 \pm 1.25$ & $0.52 \pm 0.17$ & $0.50 \pm 0.09$ \\
\hline NeuroF & $13.56 \pm 2.90$ & $29.08 \pm 8.30^{*}$ & $37.86 \pm 12.15$ & $41.72 \pm 11.23$ \\
\hline $17(R S)$-SC- $\Delta^{15}$-11-dihomo-IsoF & $4.47 \pm 1.39$ & $4.28 \pm 1.20$ & $0.39 \pm 0.18$ & $0.33 \pm 0.16$ \\
\hline
\end{tabular}

Concentration values are expressed in mean $\pm \mathrm{SD}, \mathrm{ng} / \mathrm{g}$ of tissue except ${ }^{+} \mu \mathrm{g} / \mathrm{g}$ tissue. ${ }^{*} p<0.05,{ }^{* *} p<0.01$ compared with control. Adult: $n=4$; Fetus: $n=8$. 
Table II. Level of enzymatic oxidized lipid products of AA via LOX and CYP450 pathways measured in adult and fetal brain after PFOS exposure.

\begin{tabular}{|c|c|c|c|c|}
\hline & \multicolumn{4}{|c|}{ Brain } \\
\hline & \multicolumn{2}{|c|}{ Adult } & \multicolumn{2}{|c|}{ Fetus } \\
\hline & Control & PFOS-treated & Control & PFOS-treated \\
\hline \multicolumn{5}{|c|}{ CYP450-mediated } \\
\hline $9(S)$-HETE & $13.88 \pm 2.00$ & $17.80 \pm 4.44$ & $14.16 \pm 5.37$ & $8.99 \pm 1.53^{*}$ \\
\hline 11(S)-HETE & $68.81 \pm 8.30$ & $71.88 \pm 12.28$ & $58.44 \pm 22.71$ & $40.21 \pm 10.75$ \\
\hline 20-HETE & $94.49 \pm 5.40$ & $109.65 \pm 15.74$ & $63.72 \pm 23.73$ & $47.22 \pm 11.81$ \\
\hline \multicolumn{5}{|l|}{ LOX-mediated } \\
\hline 5(S)-HETE & $39.42 \pm 12.27$ & $46.29 \pm 13.78$ & $41.87 \pm 9.31$ & $26.05 \pm 6.53^{* *}$ \\
\hline $8(S)$-HETE & $22.81 \pm 1.38$ & $28.69 \pm 7.49$ & $21.62 \pm 6.70$ & $15.49 \pm 2.55^{*}$ \\
\hline 12(S)-HETE & $113.05 \pm 12.96$ & $94.13 \pm 8.85$ & $48.55 \pm 12.56$ & $44.95 \pm 9.28$ \\
\hline 15(S)-HETE & $9.93 \pm 0.78$ & $9.56 \pm 2.39$ & $10.77 \pm 3.77$ & $9.27 \pm 2.96$ \\
\hline
\end{tabular}

Concentration values are expressed in mean $\pm \mathrm{SD}$, ng/g tissue. ${ }^{*} p<0.05,{ }^{* *} p<0.01,{ }^{* * *} p<0.001$ as compared with control. Adult: $n=4$; Fetus: $n=8$.

in this study were extracted on GD17, which is one day before term. Assuming that treated pregnant mice carry the same PFOS distribution ratio as their fetuses, we determined the representative oxidized lipid products derived from AA, EPA, DHA, and cholesterol via enzymatic and non-enzymatic pathways. The levels of non-enzymatic oxidized lipid products in PFOS-exposed fetal livers were consistently increased, but a similar trend was absent from maternal tissues. However, enzymatically oxidized lipid products derived from AA and cholesterol had a limited overall oxidation effect, with the exception of 15(S)-HETE and 27-hydroxycholesterol in fetal liver and 5(S)-HETE from maternal liver. Both 15(S)- and 5(S)-HETE are reported to have modulating roles during inflammation
[32]. Of note, $\mathrm{F}_{2}$-IsoPs $\left(15-\mathrm{F}_{2 \mathrm{t}}\right.$-IsoP and 5- $\mathrm{F}_{2 \mathrm{t}}$-IsoP) derived from AA, and the "gold" standard for in vivo oxidative damage [24-26], increased tremendously in PFOSexposed fetuses, implying that PFOS-treated fetuses were under oxidative stress. Taken together, our data suggest that PFOS affects fetal tissues more than maternal tissues. First, PFOS exposure consistently elevated total free PUFAs and cholesterol content in fetal liver. Additionally, lipid precursors were extensively and exclusively oxidized in fetal liver, which seemed to favor a non-enzymatic pathway rather than an enzymatic pathway. These results showed that PFOS-induced oxidative stress PFOS had larger effects in fetuses than in dams by releasing ROS that lead to lipid peroxidation. Subsequently, we questioned

Table III. Level of PUFAs and their non-enzymatic oxidized lipid products in adult and fetal mice kidney after PFOS treatment.

\begin{tabular}{|c|c|c|c|c|}
\hline & \multicolumn{4}{|c|}{ Kidney } \\
\hline & \multicolumn{2}{|c|}{ Adult } & \multicolumn{2}{|c|}{ Fetus } \\
\hline & Control & PFOS-treated & Control & PFOS-treated \\
\hline \multicolumn{5}{|l|}{ Lipid precursors } \\
\hline $\mathrm{AA}$ & $145.95 \pm 36.81$ & $162.86 \pm 46.79$ & $6.83 \pm 4.67$ & $24.49 \pm 4.09 * * *$ \\
\hline AdA & $0.47 \pm 0.06$ & $0.39 \pm 0.11$ & $0.18 \pm 0.11$ & $1.33 \pm 0.91^{* *}$ \\
\hline EPA & $485.09 \pm 20.60$ & $259.23 \pm 10.20^{* * *}$ & $43.72 \pm 12.36$ & $72.64 \pm 16.37 * *$ \\
\hline $\mathrm{DHA}^{+}$ & $4.69 \pm 0.27$ & $2.82 \pm 0.77^{*}$ & $0.40 \pm 0.06$ & $0.46 \pm 0.10$ \\
\hline \multicolumn{5}{|l|}{ Isoprostanoids } \\
\hline $15-\mathrm{F}_{2 \mathrm{t}}$-IsoP & $89.02 \pm 6.00$ & $114.62 \pm 6.55^{* *}$ & $12.91 \pm 11.81$ & $45.40 \pm 18.72 * * *$ \\
\hline $5-\mathrm{F}_{2 \mathrm{t}}$-IsoP $^{+}$ & $0.33 \pm 0.08$ & $0.08 \pm 0.01^{* *}$ & $0.46 \pm 0.04$ & $5.03 \pm 2.95 * * *$ \\
\hline $8-\mathrm{F}_{3 \mathrm{t}}$-IsoP & $33.04 \pm 5.82$ & $24.77 \pm 3.93$ & $39.95 \pm 6.03$ & $69.78 \pm 10.83^{* * *}$ \\
\hline $4(R S)-4-\mathrm{F}_{4 \mathrm{t}}-$ NeuroP & $68.41 \pm 5.75$ & $56.70 \pm 1.32 *$ & $9.06 \pm 8.18$ & $20.19 \pm 4.16^{* *}$ \\
\hline $\begin{array}{l}17(R S)-\mathrm{F}_{2 \mathrm{t}} \text {-dihomo-IsoP } \\
\text { Isofuranoids }\end{array}$ & $33.75 \pm 2.62$ & $22.18 \pm 2.54^{* *}$ & $7.66 \pm 6.64$ & $25.63 \pm 3.59^{* * *}$ \\
\hline IsoF & $24.51 \pm 3.71$ & $17.58 \pm 3.63$ & $6.09 \pm 5.62$ & $20.02 \pm 5.57 * * *$ \\
\hline NeuroF & $43.17 \pm 6.45$ & $50.80 \pm 8.42$ & $16.45 \pm 2.55$ & $36.29 \pm 8.88^{* * *}$ \\
\hline $\begin{array}{l}\text { 17(RS)-SC- } \Delta^{15}-11- \\
\text { dihomo-IsoF }\end{array}$ & $18.15 \pm 1.85$ & $4.54 \pm 0.73^{* * *}$ & $3.79 \pm 3.25$ & $16.15 \pm 2.65^{* * *}$ \\
\hline
\end{tabular}

Concentration values are expressed in mean $\pm \mathrm{SD}, \mathrm{ng} / \mathrm{g}$ tissue except ${ }^{+} \mu \mathrm{g} / \mathrm{g}$ tissue. ${ }^{*} p<0.05,{ }^{* *} p<0.01,{ }^{* * *} p<0.001$ compared with control. Adult: $n=4$; Fetus: $n=8$. 
Table IV. Level of enzymatic oxidized lipid products of AA via LOX and CYP450 pathways in adult and fetal kidney after PFOS exposure.

\begin{tabular}{|c|c|c|c|c|}
\hline & \multicolumn{4}{|c|}{ Kidney } \\
\hline & \multicolumn{2}{|c|}{ Adult } & \multicolumn{2}{|c|}{ Fetus } \\
\hline & Control & PFOS-treated & Control & PFOS-treated \\
\hline \multicolumn{5}{|c|}{ CYP450-mediated } \\
\hline $9(S)-\mathrm{HETE}^{+}$ & $151.86 \pm 15.60$ & $42.87 \pm 1.97 * * *$ & $51.35 \pm 10.91$ & $61.24 \pm 15.23$ \\
\hline 11(S)-HETE & $1.86 \pm 0.32$ & $0.81 \pm 0.14^{* *}$ & $2.46 \pm 0.35$ & $0.19 \pm 0.04^{* * *}$ \\
\hline 20-HETE & $3.07 \pm 0.78$ & $2.64 \pm 0.31$ & $8.22 \pm 2.39$ & $7.61 \pm 3.21$ \\
\hline \multicolumn{5}{|l|}{ LOX-mediated } \\
\hline 5(S)-HETE & $0.85 \pm 0.14$ & $3.00 \pm 0.33^{* * *}$ & $1.07 \pm 1.04$ & $0.19 \pm 0.04^{*}$ \\
\hline $8(S)$-HETE & $0.30 \pm 0.02$ & $0.09 \pm 0.01 * * *$ & $2.00 \pm 0.69$ & $1.59 \pm 1.50$ \\
\hline 12(S)-HETE & $2.44 \pm 0.58$ & $0.36 \pm 0.01^{* *}$ & $15.80 \pm 2.03$ & $0.02 \pm 0.01$ \\
\hline 15(S)-HETE ${ }^{+}$ & $74.79 \pm 21.20$ & $266.66 \pm 51.73^{* *}$ & $3.84 \pm 1.77$ & $11.99 \pm 7.02 * *$ \\
\hline
\end{tabular}

Concentration values are expressed in mean $\pm \mathrm{SD}, \mu \mathrm{g} / \mathrm{g}$ tissue except ${ }^{+} \mathrm{ng} / \mathrm{g}$ tissue. ${ }^{*} p<0.05,{ }^{* *} p<0.01,{ }^{* * *} p<0.001$ compared with control. Adult: $n=4$; Fetus: $n=8$.

whether the PUFAs were oxidized in maternal tissues and transported to the fetus, or the accumulated PFOS within fetal tissues led to lipid peroxidation. The fact that the oxidized lipid products in maternal tissues showed insignificant differences in the presence and absence of PFOS eliminates the possibility that these oxidized products were transferred to the fetuses. Thus, we can assume that the elevation of lipid peroxidation in fetal livers was due to the presence of PFOS in the tissue.

The exclusive lipid peroxidation in fetal livers then led us to postulate that the maturity of fetal antioxidant system is not in place during early stages of fetus development, or it led to antioxidant system dysfunction that resulted in a high level of oxidative stress. It is noted that, depending on the species and organs, glutathione and antioxidant enzymes, such as SOD, CAT and glutathione peroxidase, are equally important to fetal development [26]. Regardless, SOD and CAT antioxidant enzyme activities were measured in maternal liver and placenta to test our hypothesis. Surprisingly, no significant differences were observed in the level of each antioxidant enzyme in the presence of PFOS in both types of tissues. However, because of the sample limitations in fetuses, we remain inconclusive on whether the excess lipid peroxidation experienced in fetal tissues is due to the maturity of their antioxidant systems.

Furthermore, a recent study reports on the formation of free radicals from aqueous PFOS in the presence of iron under ultraviolet (UV) irradiation [33]. Although this is an in vitro study, reactive PFOS radicals are formed with the assistance of an exogenous UV source. High amounts of free iron are present in the fetus during pregnancy, and iron is known to facilitate lipid peroxidation, particularly through the non-enzymatic free radial reaction, to generate hydrogen peroxide, superoxide radical, and hydroxyl radical [34]. Hence, the high level of iron in developing fetuses could be a main culprit for the free radical generation in the presence of PFOS presented here.
In a separate study, we analyzed the brain and kidney from adult female mice exposed to the same level of PFOS, which were then compared with the fetal organs. Adult and fetal mice exposed to PFOS showed elevated levels of $\mathrm{F}_{2}$-IsoPs in kidney, indicating the presence of oxidative stress. The high levels of oxidative damage found in the fetal kidney may have detrimental effects on developmental because $\mathrm{F}_{2}$-IsoPs is also reported to be an endogenous vasoconstrictor $[24,25]$. In addition to $\mathrm{F}_{2}{ }^{-}$ IsoPs, levels of oxidized lipid products derived from other precursors were increased in both adult and fetal kidney. Most notably, the isofuranoid group (IsoFs, NeuroFs, and $17(R S)$-SC- $\Delta^{15}$-11-dihomo-IsoF), which is generated under high oxygen tension, was significantly augmented by PFOS treatment compared with the control. The importance of the endogenous isofuranoids is unknown, but it appears to be a potentially more stable form of oxidized lipid products, in particular for the brain [24,35,36]. Nevertheless, unlike the kidney, the mouse brain was not consistently affected, possibly because limited PFOS was taken up in the brain [37], which might have been potentially hindered by the blood-brain barrier. Although brain development matures at a much later stage than many other organs, the blood-brain barrier is a natural protector and is fully developed before birth [38].

It should be noted that this is the first study to measure the precise oxidized lipid products of the non-enzymatic pathway, particularly $17(R S)$-SC- $\Delta^{15}-11$-dihomo-IsoF, in mouse brain tissues. Recent measurements on 17(RS)-SC$\Delta^{15}$-11-dihomo-IsoF levels were performed on pig and rat brain samples [35,36], which have distinctly different results from our data. However, the species used, the maturity of the subjects, the region of the brain assessed (as whole brain was used in this study), and the sample sizes used might lead to the inconsistencies with the current result. Taken together, PFOS-induced lipid peroxidation seemed to affect tissues in the order of kidney $>$ liver $>$ brain. 
There are previous reports on the effects of prenatal PFOS exposure. Notwithstanding this, samples from these studies were mainly conducted at birth or postnatal stages. Additionally, the PFOS concentrations in the exposed dams were shown to increase steadily throughout gestation and postnatal stages $[13,16]$. However, the amount of PFOS found in the pups was only measured at postnatal days, although they were exposed during the prenatal period. Therefore, PFOS levels were not previously investigated in paired dams and pups during gestation. To the best of our knowledge, this is the first report that studies trans-generational effects of PFOS in mouse maternalfetal lipid metabolism during gestation.

In conclusion, we have shown, for the first time, that PFOS exposure in pregnant mice induces notable lipid peroxidation in fetuses during their gestation. However, PFOS-induced fetal lipid peroxidation is organ-specific, and occurs primarily through the non-enzymatic lipid peroxidation pathway. Nevertheless, the detrimental effects of prolonged oxidative stress during gestation are not determined here. Therefore, a postnatal study needs to be employed to identify whether the effects on fetal development will continue at a later age due to altered lipid metabolism and if cognitive behaviors are affected.

\section{Acknowledgements}

This work was supported by General Research Fund (HKBU261812) and the University of Hong Kong Seed Funding Programme for Basic Research (No. 104002391 and 104003173).

\section{Declaration of interest}

The authors report no declarations of interest. The authors alone are responsible for the content and writing of the paper.

\section{References}

[1] Giesy JP, Kannan K. Perfluorochemical surfactants in the environment. Environ Sci Technol 2002;36:146A-152A.

[2] Olsen GW, Burris JM, Ehresman DJ, Froehlich JW, Seacat AM, Butenhoff JL, Zobel LR. Half-life of serum elimination of perfluorooctanesulfonate,perfluorohexanesulfonate, and perfluorooctanoate in retired fluorochemical production workers. Environ Health Perspect 2007;115:1298-1305.

[3] Giesy JP, Kannan K. Global distribution of perfluorooctane sulfonate in wildlife. Environ Sci Technol 2001;35:1339-1342.

[4] Martin JW, Smithwick MM, Braune BM, Hoekstra PF, Muir DC, Mabury SA. Identification of long-chain perfluorinated acids in biota from the Canadian Arctic. Environ Sci Technol 2004;38:373-380.

[5] Chang SC, Noker PE, Gorman GS, Gibson SJ, Hart JA, Ehresman DJ, Butenhoff JL. Comparative pharmacokinetics of perfluorooctanesulfonate (PFOS) in rats, mice, and monkeys. Reprod Toxicol 2012;33:428-440.

[6] Wang Y, Fu J, Wang T, Liang Y, Pan Y, Cai Y, Jiang G. Distribution of perfluorooctane sulfonate and other perfluorochemicals in the ambient environment around a manufacturing facility in China. Environ Sci Technol 2010;44:8062-8067.

[7] Kim S, Choi K, Ji K, Seo J, Kho Y, Park J, et al. Trans-placental transfer of thirteen perfluorinated compounds and relations with fetal thyroid hormones. Environ Sci Technol 2011;45:7465-7472.

[8] Wan HT, Zhao YG, Leung PY, Wong CK. Perinatal exposure to perfluorooctane sulfonate affects glucose metabolism in adult offspring. PLoS One 2014;9:e87137.

[9] Wan HT, Zhao YG, Wei X, Hui KY, Giesy JP, Wong CK. PFOS-induced hepatic steatosis, the mechanistic actions on beta-oxidation and lipid transport. Biochim Biophys Acta 2012;1820:1092-1101.

[10] Wang L, Wang Y, Liang Y, Li J, Liu Y, Zhang J, Zhang A. PFOS induced lipid metabolism disturbances in BALB/c mice through inhibition of low density lipoproteins excretion. Sci Rep 2014;4:4582.

[11] Onishchenko N, Fischer C, Wan Ibrahim WN, Negri S, Spulber S, Cottica D, Ceccatelli S. Prenatal exposure to PFOS or PFOA alters motor function in mice in a sex-related manner. Neurotox Res 2011;19:452-461.

[12] Bjork JA, Wallace KB. Structure-activity relationships and human relevance for perfluoroalkyl acid-induced transcriptional activation of peroxisome proliferation in liver cell cultures. Toxicol Sci 2009;111:89-99.

[13] Thibodeaux JR, Hanson RG, Rogers JM, Grey BE, Barbee $\mathrm{BD}$, Richards JH, et al. Exposure to perfluorooctane sulfonate during pregnancy in rat and mouse. I: maternal and prenatal evaluations. Toxicol Sci 2003;74:369-381.

[14] Liu C, Yu K, Shi X, Wang J, Lam PK, Wu RS, Zhou B. Induction of oxidative stress and apoptosis by PFOS and PFOA in primary cultured hepatocytes of freshwater tilapia (Oreochromis niloticus). Aquat Toxicol 2007;82:135-143.

[15] Shi X, Zhou B. The role of Nrf2 and MAPK pathways in PFOS-induced oxidative stress in zebrafish embryos. Toxicol Sci 2010;115:391-400.

[16] Lau C, Thibodeaux JR, Hanson RG, Rogers JM, Grey BE, Stanton ME, et al. Exposure to perfluorooctane sulfonate during pregnancy in rat and mouse. II: postnatal evaluation. Toxicol Sci 2003;74:382-392.

[17] Wan HT, Leung PY, Zhao YG, Wei X, Wong MH, Wong CK. Blood plasma concentrations of endocrine disrupting chemicals in Hong Kong populations. J Hazard Mater 2013;261: 763-769.

[18] Zhao YG, Wan HT, Law AY, Wei X, Huang YQ, Giesy JP, et al. Risk assessment for human consumption of perfluorinated compound-contaminated freshwater and marine fish from Hong Kong and Xiamen. Chemosphere 2011;85: 277-283.

[19] Chung ML, Lee KY, Lee CY. Profiling of oxidized lipid products of marine fish under acute oxidative stress. Food Chem Toxicol 2013;53:205-213.

[20] Lee CY, Huang SH, Jenner AM, Halliwell B. Measurement of F2-isoprostanes, hydroxyeicosatetraenoic products, and oxysterols from a single plasma sample. Free Radic Biol Med 2008;44:1314-1322.

[21] Lee CY, Seet RC, Huang SH, Long LH, Halliwell B. Different patterns of oxidized lipid products in plasma and urine of dengue fever, stroke, and Parkinson's disease patients: cautions in the use of biomarkers of oxidative stress. Antioxid Redox Signal 2009;11:407-420.

[22] Leung KS, Chen X, Zhong W, Yu AC, Lee CY. Microbubblemediated sonoporation amplified lipid peroxidation of Jurkat cells. Chem Phys Lipids 2014;180:53-60.

[23] Shui G, Cheong WF, Jappar IA, Hoi A, Xue Y, Fernandis AZ, et al. Derivatization-independent cholesterol analysis in crude lipid extracts by liquid chromatography/mass spectrometry: applications to a rabbit model for atherosclerosis. J Chromatogr A 2011;1218:4357-4365.

[24] Morrow JD, Hill KE, Burk RF, Nammour TM, Badr KF, Roberts LJ, 2nd. A series of prostaglandin F2-like compounds 
are produced in vivo in humans by a non-cyclooxygenase, free radical-catalyzed mechanism. Proc Natl Acad Sci U S A 1990;87:9383-9387.

[25] Galano JM, Lee JC, Gladine C, Comte B, Le Guennec JY, Oger C, Durand T. Non-enzymatic cyclic oxygenated metabolites of adrenic, docosahexaenoic, eicosapentaenoic and $\alpha$-linolenic acids; bioactivities and potential use as biomarkers. Biochim Biphysic Acta 2015;1851:446-455.

[26] Halliwell B, Gutteridge J. 2007. Free radicals in biology and medicine. 4th ed. United Kingdom: Oxford.

[27] Martin MT, Brennan RJ, Hu W, Ayanoglu E, Lau C, Ren H, et al. Toxicogenomic study of triazole fungicides and perfluoroalkyl acids in rat livers predicts toxicity and categorizes chemicals based on mechanisms of toxicity. Toxicol Sci 2007;97:595-613

[28] Kennedy GL Jr, Butenhoff JL, Olsen GW, O'Connor JC, Seacat AM, Perkins RG, et al. The toxicology of perfluorooctanoate. Crit Rev Toxicol 2004;34:351-384.

[29] Arterburn LM, Hall EB, Oken H. Distribution, interconversion, and dose response of $\mathrm{n}-3$ fatty acids in humans. Am J Clin Nutr 2006;83:1467S-1476S.

[30] Luebker DJ, York RG, Hansen KJ, Moore JA, Butenhoff JL. Neonatal mortality from in utero exposure to perfluorooctanesulfonate (PFOS) in Sprague-Dawley rats: dose-response, and biochemical and pharamacokinetic parameters. Toxicology 2005;215:149-169.

[31] Monroy R, Morrison K, Teo K, Atkinson S, Kubwabo C, Stewart B, Foster WG. Serum levels of perfluoroalkyl

\section{Supplementary material available online}

Supplementary Table I to be found at online http://informahealthcare.com/doi/abs/10.3109/10715762.2015.1027199. compounds in human maternal and umbilical cord blood samples. Environ Res 2008;108:56-62.

[32] Levy BD, Clish CB, Schmidt B, Gronert K, Serhan CN. Lipid mediator class switching during acute inflammation: signals in resolution. Nat Immunol 2001;2:612-619.

[33] Jin L, Zhang P, Shao T, Zhao S. Ferric ion mediated photodecomposition of aqueous perfluorooctane sulfonate (PFOS) under UV irradiation and its mechanism. J Hazard Mater 2014;271:9-15.

[34] Halliwell B, Gutteridge JM. Oxygen free radicals and iron in relation to biology and medicine: some problems and concepts. Arch Biochem Biophys 1986;246:501-514.

[35] de La Torre A, Lee YY, Oger C, Sangild PT, Durand T, Lee JC, Galano JM. Synthesis, discovery, and quantitation of dihomo-isofurans: biomarkers for in vivo adrenic Acid peroxidation. Angew Chem Int Ed Engl 2014;53:6249-6252.

[36] de la Torre A, Lee YY, Mazzoni A, Guy A, Bultel-Ponce V, Durand T, et al. Total Syntheses and In Vivo Quantitation of Novel Neurofuran and Dihomo-isofuran Derived from Docosahexaenoic Acid and Adrenic Acid. Chemistry 2015;21: 2442-2446.

[37] Borg D, Bogdanska J, Sundstrom M, Nobel S, Hakansson H, Bergman A, et al. Tissue distribution of (35)S-labelled perfluorooctane sulfonate (PFOS) in C57B1/6 mice following late gestational exposure. Reprod Toxicol 2010;30:558-565.

[38] Saunders NR, Liddelow SA, Dziegielewska KM. Barrier mechanisms in the developing brain. Front Pharmacol 2012;3:46. 